\title{
Intravenous immunoglobulin-associated renal failure in a patient with post-transfusion purpura
}

\author{
Sujith V. Cherian, Subhraleena Das, Amarinder S. Garcha, Divey Manocha, Nitish Kosaraju \\ From the Department of Internal Medicine, SUNY Upstate Medical University, Syracuse, New York, USA \\ Correspondence: Sujith V. Cherian · 50 Presidential Plaza, Apt 2106, Syracuse, New York, USA 13202 ·T: 1818854 5737, F: 13154644484 \\ ·sujithvcherian@gmail.com \\ Ann Saudi Med 2012; 32(6): 646-648
}

DOI: $10.5144 / 0256-4947.2012 .01 .7 .1500$

\begin{abstract}
Intravenous immunoglobulin (IVIG), initially developed for immunodeficiency disorders, has now been used for multiple autoimmune diseases and infections. These are generally well tolerated, with few adverse effects. Acute kidney injury has been described in very rare instances. We report an interesting case of a 59-year-old African American male with a pertinent history of diabetes mellitus, hypertension, endocarditis, and peripheral vascular disease, who was diagnosed with post-transfusion purpura. He was then treated with IVIG and subsequently developed an acute worsening of renal function in a time span of 3 days. The etiology remained elusive even after an extensive workup. Renal biopsy was done finally, which showed findings suggestive of osmotic nephropathy that was traced to the sucrose used as a stabilizing agent in the IVIG. In light of the increasing use of IVIG, it is therefore highly recommended that clinicians are well aware of this side effect of IVIG.
\end{abstract}

$\mathrm{I}$ ntravenous immunoglobulin (IVIG) is a highly purified immunoglobulin $G$ preparation made from pooled human plasma. It is usually prescribed for a variety of conditions that include hematological, autoimmune, and infectious diseases. The initial use of IVIG was hampered by the large incidence of adverse effects like chills, back pain, and fever. This was largely believed to be secondary to the immune complexes activating the complement system. Stabilization with products like sucrose, maltose, glycine, sorbitol, or albumin helped avert these side effects. Renal failure has been reported as a rare complication accounting for $<1 \%$ cases of the IVIG therapy.

\section{CASE}

A 59-year-old African American male with a medical history significant for hypertension, diabetes mellitus, and extensive peripheral vascular disease was admitted with left toe gangrene and complete occlusion of the femoropopliteal bypass graft. The patient underwent an amputation of the left knee. He received 2 units of packed red blood cells (PRBCs) because of the blood loss related to the procedure. His postoperative course was complicated with fevers and methicillin-resistant Staphylococcus aureus (MRSA) bacteremia and aortic valve endocarditis. He was started on intravenous vancomycin therapy and discharged. Two days later, he was admitted with complaints of persistent fevers despite being on vancomycin. The physical examination at the time of admission revealed that the patient was febrile with a temperature of $101.4^{\circ} \mathrm{F}$. The systemic examination did not seem to show any obvious source of infection. The diagnostic workup included routine labs, blood and urine cultures, and $x$-rays. These were all negative except for a platelet count of $18 \times 10^{9} / \mathrm{L}$ (reference range, $150-400 \times 10^{9} / \mathrm{L}$ ). These dropped to $4 \times 10^{9} / \mathrm{L}$ by day 3 with no evidence of any spontaneous bleed. Heparin-induced thrombocytopenia (recent use of heparin), endocarditis, and vancomycin-related side effects were considered among other differentials. Withholding heparin and vancomycin did not improve the clinical picture. We also kept the possibility of post-transfusion purpura in view of the recent history of transfusion of PRBCs. His results revealed negative HIT antibody and positive human platelet alloantigen antibody (anti-HPA-1a) to confirm the diagnosis of 


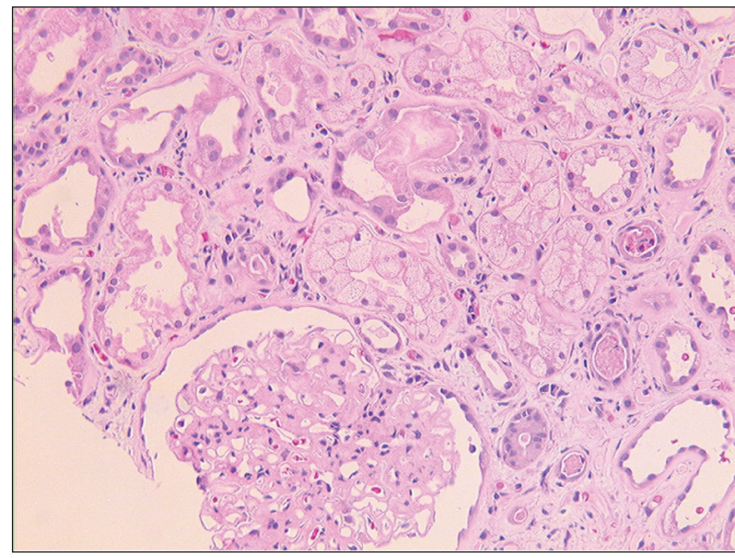

Figure 1. Vacuolization and swelling of the proximal tubule consistent with osmotic nephropathy $(H \& E, \times 100)$.

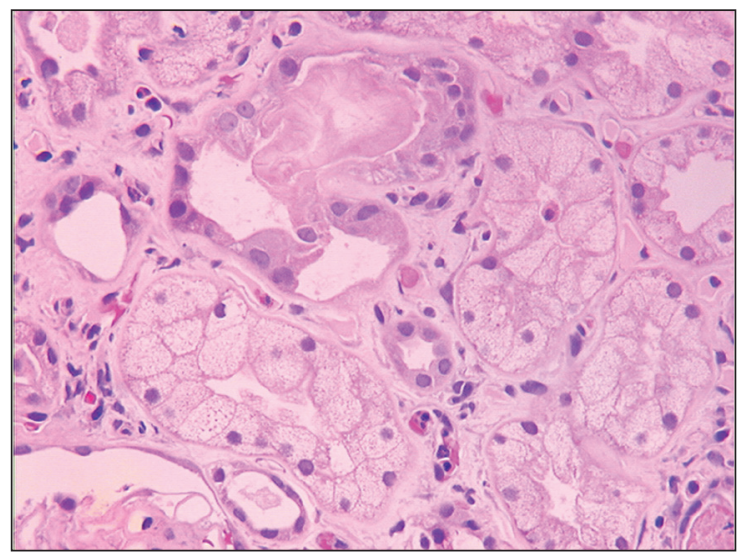

Figure 2. H\&E stained specimen showing proximal tubular vacuolization $(\times 400)$.

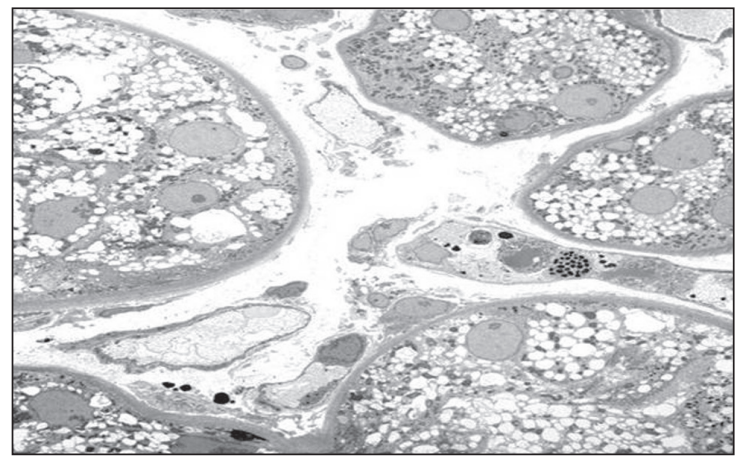

Figure 3. Electron microscopic picture showing osmotic nephropathy.

post-transfusion purpura. He was immediately started on a high dose of IVIG (Carimune NF ZLB Behring AG, Berne, Switzerland) with subsequent improvement in the platelet count. Unfortunately, within 3 days of starting IVIG, the patient showed worsening kidney function. Serum creatinine increased from 1.4 to 6.6 $\mathrm{mg} / \mathrm{dL}$ (reference range, $0.4-1.1 \mathrm{mg} / \mathrm{dL}$ ) with oliguria. The underlying cause of this renal failure was still elusive even after a detailed workup. Considering the timeline of onset of renal failure with the administration of IVIG, the diagnosis of IVIG-induced nephropathy was entertained among differential diagnoses. The diagnostic workup ended in a renal biopsy (Figures 1-3) that showed findings suggestive of osmotic nephropathy, which was traced to the sucrose used as stabilizing agent in the IVIG. The IVIG was withdrawn. His renal function and urine output improved to baseline over the next 7 to 10 days.

\section{DISCUSSIONS}

Renal failure has been reported as a rare complication accounting for $<1 \%$ cases of IVIG therapy. Only few case reports are available that describe this entity. The analysis of previous case reports suggest that renal adverse effects occurred in the first 10 days after initiation of therapy, with a mean of 3 days and a peak of creatinine around day 5. To make a diagnosis, a temporal association has to be established with the administration of IVIG. A clinical diagnosis is made after ruling out other causes and is confirmed with renal biopsy.,3

It has been suggested that the mechanism underlying the IVIG-induced renal failure is a result of the effect of the osmotic load posed to the proximal tubules by the sucrose content of the IVIG preparation. The absence of the sucrase enzyme at the brush border of the proximal tubular cells results in high concentrations of sucrosethat eventually result in pinocytosis of sucrose into the cells. This is followed by water and causes it to swell resulting in cytoplasmic vacuolization and degeneration of proximal tubular cells. ${ }^{4,5}$ This is reiterated by the fact that most of the case reports (90\%) in an FDA report and $78 \%$ in a review of the published studies by Orbach et al of IVIG nephropathy involved sucrose as the stabilizing agent. Of note, the incidence of renal failure when maltose was used as the stabilizing agent was also very low, probably secondary to the fact that the maltase enzyme is present in the brush border of proximal tubular epithelial cells. ${ }^{6}$ Furthermore, the findings in biopsy results are identical to those seen in human beings and animals with sucrose nephropathy. Other mechanisms have also been suggested for the osmotic injury caused (a) by enhancing the tubuloglomerular feedback resulting in afferent arteriolar constriction and a decline in filtration pressure and (b) severe renal artery constriction causing a decrease in glomerular filtration rate. ${ }^{7}$ 
In reviewing the course of ARF after IVIG, most cases resolved spontaneously. About $36 \%$ of the patients required hemodialysis. The duration of renal failure was between 3 to 45 days. The average duration was 13 days with the creatinine usually reaching the baseline in 7 to 15 days. ${ }^{8}$ Some of the risk factors for developing the IVIG-induced renal failure include increased age ( $>65$ years), impaired renal function at baseline, dehydration, high-dose IVIG therapy (400-2000 mg/ $\mathrm{kg}$ ), use of sucrose stabilizer, and the rate at which the IVIG is administered. Diabetes mellitus, diuretic use, and concomitant nephrotoxic drug administration have been also cited as risk factors. ${ }^{3-5,7}$

Our patient showed onset of renal failure by the third day, with the creatinine peaking on the eighth day followed by a return to baseline renal function within 15 days. The IVIG used was Carimune NF (1.67 g sucrose/g protein). Even though his age was 59 years, which is slightly below the average age group (generally $>65$ years age), he had other risk factors such as impaired renal function, diabetes mellitus, and the use of sucrose stabilizers in the IVIG administered. Fortunately, his renal function improved spontaneously without the need for hemodialysis. Of note, Carimune NF or Sandoglobulin (ZLB Behring AG, Berne, Switzerland) has the highest sucrose content among the conventional IVIGs used. As in the other cases described, biopsy findings were consistent with osmotic injury similar to sucrose nephropathy. ${ }^{7}$

To summarize, in general, recommendations are to use sucrose-containing IVIG preparations with caution and switch to other agents when risk factors are present. Adequate hydration along with avoidance of other nephrotoxic medications and diuretics is recommended. Using the smallest dose possible as well as prolonging the infusion interval also helps minimize the brunt of renal injury caused.

\section{REFERENCES}

1. Daphnis E, Stylianou K, Alexandrakis M, Xylouri I, Vardaki E, Stratigis $S$, et al. Acute renal failure, translocational hyponatremia and hyperkalemia following intravenous immunoglobulin therapy. Nephron Clin Pract 2007;106:c143-8.

2. Graumann A, Zawada ET Jr. Acute renal failure after administering intravenous immunoglobulin. Postgrad Med 2010;122:142-7.

3. Orbach H, Tishler M, Shoenfeld Y. Intravenous immunoglobulin and the kidney-a two-edged sword. Semin Arthritis Rheum 2004:34:593-601. 4. Ahsan N, Palmer BF, Wheeler D, Greenlee $\mathrm{RG} \mathrm{Jr}$, Toto RD. Intravenous immunoglobulin induced osmotic nephrosis. Arch Intern Med 1994;154:1985-7.

5. Dickenmann M, Oett T, Mihatsch MJ. Osmotic nephrosis: Acute kidney injury with accumulation of proximal tubular lysosomes due to administration of exogenous solutes. Am J Kidney Dis 2008;51:491-503.

6. Kwan TH, Tong MK, Siu YP, Leung KT, Lee HK,
Yung $\mathrm{CY}$, et al. Acute renal failure related to intravenous immunoglobulin infusion in an elderly woman, Hong Kong Med J 2005:11:45-9.

7. Haskin JA, Warner DJ, Blank DU. Acute renal failure after large doses of intravenous immunoglobulin. Ann Pharmacother 1999;33:800-3.

8. Orbach H, Katz U, Sherer Y, Shoenfeld Y. Intravenous immunoglobulin: adverse effects and safe administration. Clin Rev Allergy Immunol 2005:29:173-84. 